\title{
Pulmonem et pectus
}

\author{
Richard K. Freeman, MD, MBA \\ From the Department of Thoracic and Cardiovascular Surgery, St Vincent Hospital, Indianapolis, Ind. \\ Disclosures: Author has nothing to disclose with regard to commercial support. \\ Received for publication Feb 13, 2018; accepted for publication Feb 15, 2018. \\ Address for reprints: Richard K. Freeman, MD, MBA, 8433 Harcourt Rd, Indianapolis, IN 46260 (E-mail: \\ Richard.Freeman@ascension.org). \\ J Thorac Cardiovasc Surg 2018;155:2148-9 \\ $0022-5223 / \$ 36.00$ \\ Copyright $($ c 2018 Published by Elsevier Inc. on behalf of The American Association for Thoracic Surgery \\ https://doi.org/10.1016/j.jtcvs.2018.02.040
}

Significant advances in the diagnosis and treatment of lung cancer have been made in the last 2 decades, providing hope that the most common cause of cancer-related death in men and women in the United States ${ }^{1}$ will be recognized earlier and treated more effectively. Computed tomographic (CT) imaging screening for high-risk populations is a direct result of such efforts. These screening studies, along with other imaging protocols, such as coronary calcium score CT scans and the increased use of CT imaging for abdominal and thoracic complaints, have all resulted in a drastic increase in the number of patients found to have an abnormality of the pulmonary parenchyma. The potential morbidity and cost incurred in evaluating these abnormalities, most of which will prove to be benign, are important considerations in the discussion of lung cancer screening.

Within the larger discussion of pulmonary nodule evaluation is the unique category of centrally located or nonpalpable parenchymal lung lesions. Multiple techniques have been evaluated to assist in the evaluation of such findings. As summarized in the article in this issue of the Journal by Thistlethwaite and colleagues, ${ }^{2}$ none of these techniques has yet found the mark of accuracy, reasonable cost, convenience for the patient and the surgical team, and safety.

The investigation of Thistlethwaite and colleagues ${ }^{2}$ combines 2 techniques from different categories of localization: visual localization with methylene blue and double wire localization. This provides a rescue opportunity in the case of failure of either the visual or mechanical localization component. Their use of a multidisciplinary review of patients before making a decision regarding the appropriateness of localization is also to be commended and has been shown to conserve resources and maximize patient safety. ${ }^{3}$

By implementing this investigation's localization protocol, Thistlethwaite and colleagues ${ }^{2}$ were able to achieve a diagnostic success rate of $96.8 \%$ in a large series of

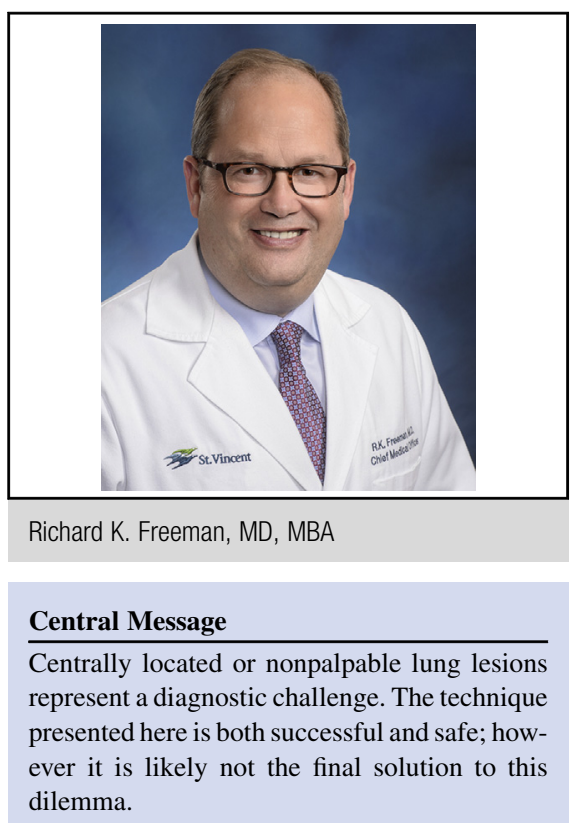

See Article page 2140 .

patients. Minimal complications were also reported. The technique still exposes patients, however, to some risk and additional cost. Although the data do not allow specific comment on a learning curve, the ability to disseminate this technique also remains to be seen.

Ultimately, the technique so proficiently implemented by Thistlethwaite and colleagues ${ }^{2}$ is likely not the ultimate solution to this diagnostic problem. Carcinomas of the lung and the breast, pulmonem et pectus in Latin, share many parallels. Interestingly, the projected reduction in mortality from lung cancer produced by implementing CT screening is $20 \%$ - exactly the reduction seen with mammography for invasive carcinoma of the breast. It is no coincidence that the mechanical localization technique of image-guided wire placement in this report is borrowed from the realm of breast cancer diagnosis as well. It therefore would not be surprising to discover that the ultimate answer to the problem of localizing central or nonpalpable lung lesions is analogous to the modality that has become the preferred breast cancer diagnostic technique because it is percutaneous, highly accurate, and rapid: stereotactic biopsy. Until such a technique is developed for the lung, however, the work of Thistlethwaite and colleagues ${ }^{2}$ will serve as a standard of accuracy and safety. 


\section{References}

1. Surveillance, Epidemiology, and End Results (SEER) Program. SEER*Stat Database: Mortality-All COD, Aggregated With State, Total U.S. (1969-2014) $<$ Katrina/Rita Population Adjustment>, National Cancer Institute, DCCPS, Surveillance Research Program, released December 2016. Underlying mortality data provided by NCHS (www.cdc.gov/nchs). Available at: http://www.seer.cancer.gov.
2. Thistlethwaite PA, Gower JR, Hernandez M, Zhang Y, Picel AC, Roberts AC. Needle localization of small pulmonary nodules: lessons learned. J Thorac Cardiovasc Surg. 2018;155:2140-7.

3. Freeman RK, Van Woerkom J, Vyverberg A, Ascioti AJ. The effect of a multidis ciplinary thoracic malignancy conference on the treatment of patients with lung cancer. Eur J Cardiothorac Surg. 2010;38:1-5. 